\title{
Conception innovante de charnière de volet d'airbag frontal passager
}

\author{
GRÉGORY SPINGLER ${ }^{\mathrm{a}}$ \\ Crash et Sécurité Habitacle, Visteon Systèmes Intérieurs, Centre Technique, Rue Léon Duhamel, BP 87, 62440 Harnes, France
}

Reçu le 15 juillet 2008

\begin{abstract}
Résumé - Lors du développement d'un nouveau véhicule, une phase importante de l'aspect sécurité concerne le dimensionnement de la planche de bord et en particulier de la zone airbag passager. En phase prototype, afin de valider les conceptions, des essais expérimentaux de déploiement airbag sont menés. Parfois, des projections de matière, particulièrement agressives pour les occupants du véhicule, peuvent survenir amenant à la non-conformité du produit. Ces projections peuvent provenir de l'arrachement de matière lors de l'ouverture du volet d'airbag mais aussi de la fracture de celui-ci lors de son impact contre le pare-brise ou la planche de bord. L'objectif de l'étude consiste à proposer un nouveau type de charnière métallique liant le volet à la planche de bord afin de freiner le volet avant son impact avec une autre pièce de l'habitacle. La démarche employée se base sur des conceptions existantes et utilise les codes de calcul éléments-finis afin de déterminer une solution plus robuste, satisfaisant l'ensemble des spécifications croissantes imposées par les constructeurs. La meilleure conception est ensuite prototypée afin de valider expérimentalement la viabilité du produit planche de bord en terme de sécurité habitacle.
\end{abstract}

Mots clés : Sécurité automobile / airbag passager / conception / planche de bord / simulation numérique

\begin{abstract}
New passenger airbag door hinge design for front impact. For new automotive development, the design of instrument panels must be adjusted to Car Manufacturers' specifications. Safety concept has to be ensured by a correct design of airbag area. To validate solutions, prototype airbag deployment tests are made, and sometimes, parts can break and let pieces fly directly to occupant direction at extreme velocities. These fractures are mainly due to the impact of the airbag flap against the windscreen or the instrument panel. To meet the safety standards, this paper deals with a new shape of metallic hinge between the passenger airbag door and the instrument panel. A brake function, integrated into the hinge, is presented, and the final study based on numerical design with FE codes shows a new design that avoids any impact of the passenger airbag door with other parts of the cockpit. At last, this best solution is prototyped and validated with physical tests; that contributes to passive safety improvement.
\end{abstract}

Key words: Automotive safety / passenger airbag / instrument / design panel / numerical simulation

\section{Introduction}

Depuis les années 90, un axe de développement fort a été mené dans la conception des véhicules par l'intégration de la notion de sécurité passive. Au niveau de la sécurité habitacle, le couple airbag/ceinture constitue sans aucun doute l'avancée technologique la plus pertinente. L'airbag voit, à présent, ses applications décupler au sein du véhicule. Afin de parfaire l'objectif principal de retenue, la conception des planches de bord accueillant l'airbag passager avant doit être correctement dimensionnée de façon à garantir un déploiement de sac conforme aux cahiers des charges

\footnotetext{
${ }^{a}$ Auteur pour correspondance :

gspingle@visteon.com
}

établis par les constructeurs. Visteon Systèmes Intérieurs, équipementier mondial leader dans les domaines de la planche de bord et du panneau de porte, développe des conceptions avancées en vue de répondre favorablement aux attentes clients. Les spécifications primordiales liées à la planche de bord se résument à une ouverture du volet de planche de bord idéalement $7 \mathrm{~ms}$ après la mise à feu de la cartouche pyrotechnique sans cassure ni éjection de pièces ou parties de pièce dans une gamme de température allant de $-35^{\circ} \mathrm{C}$ à $+85^{\circ} \mathrm{C}$.

L'un des meilleurs systèmes consiste à décomposer la phase d'ouverture du volet en trois étapes :

- la première étape a pour fonction de dégager le volet de la planche de bord, ce qui s'opère, en général, par un mouvement de translation suivant $Z$; 
a)

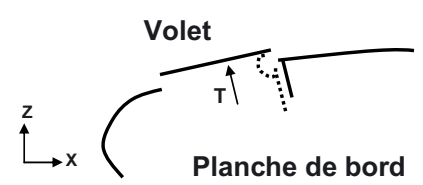

b)

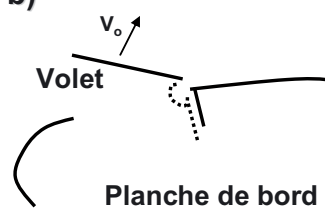

c)

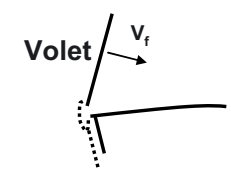

Planche de bord

Fig. 1. Schématisation de la zone airbag passager.

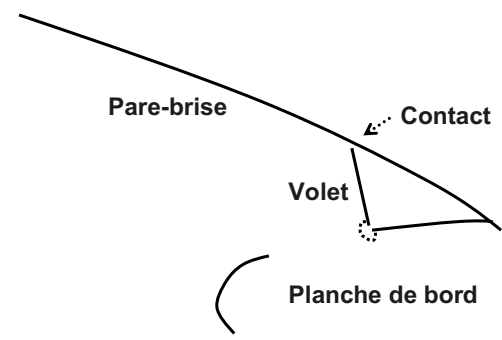

(a)

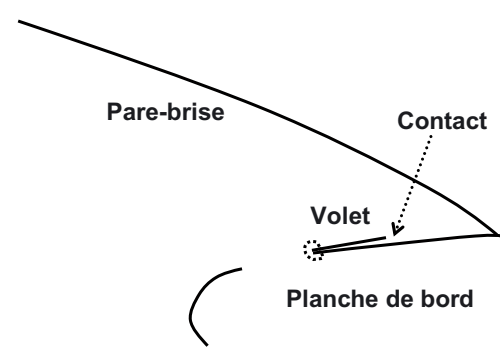

(b)

Fig. 2. Schématisation des configurations de contact volet. a) Contact volet/pare-brise Airbag en zone arrière. b) Contact volet/planche de bord Airbag en zone avant.

- la seconde étape a pour objectif d'assurer le mouvement de rotation du volet autour de son axe charnière avec la planche de bord. Cette fonction se retrouve dans toutes les conceptions puisqu'elle autorise le déploiement du sac dans les meilleurs délais;

- la troisième et dernière étape consiste à freiner le volet mis en vitesse par la pression du sac. La fonction frein, objet de cet article, a pour objet principal d'éviter le contact violent entre l'extrémité du volet et la planche de bord ou le pare-brise.

La cinématique d'ouverture volet est représentée sur la figure 1.

Lors d'un déploiement airbag, le volet s'ouvre sous la pression du sac suivant le mouvement de translation noté $T$, atteint une vitesse tangentielle en extrémité de volet, $V_{\mathrm{o}}$, de l'ordre de $100 \mathrm{~m} . \mathrm{s}^{-1}$, avant de décélérer grâce à des mécanismes prévus à cet effet, encore appelés « charnières », pour atteindre une vitesse minimale $V_{\mathrm{f}}$.

Ces charnières peuvent se présenter sous différentes formes (métallique, composite, polymère, élastomère, sangle, ... ) et ont communément pour premier objectif de garantir la tenue planche de bord/volet. Le second objectif, objet de cette étude, consiste à ralentir et/ou arrêter le volet avant son contact avec la planche de bord ou le pare-brise, comme représenté sur la figure 2.

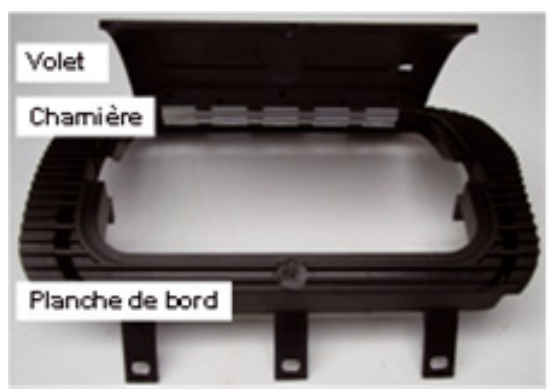

Fig. 3. Visualisation de la conception de volet airbag.

L'approche discutée dans cet article touche plus particulièrement la conception de l'articulation liant le volet airbag à la planche de bord. L'objectif consiste à définir un concept permettant de décélérer le volet, sur la base d'un produit existant constitué d'une tôle métallique. La conception actuelle, représentée sur la figure 3, s'appuie sur une préforme en double $\mathrm{U}$, ajourée localement, et surmoulée en ses deux points d'ancrage par un matériau « renfort » chargé à $30 \%$ de fibres de verre. Ces renforts soudés à la planche de bord ont donc été intégrés afin de garantir la conformité du produit suite au contact volet/pare-brise, mais l'objectif de masse et de coût a dû être dégradé.

\section{Démarche}

Le but final de la conception est la recherche d'une solution charnière suffisamment dimensionnée pour éviter tout contact du volet une fois ouvert. L'intégration de la notion de frein intervient alors au niveau du concept. Pour ce faire, l'utilisation de codes de calcul de crash, tels que Pam-Crash ${ }^{\mathrm{TM}}$ [1] est un atout essentiel dans le développement rapide de solutions mécaniques répondant à nos spécifications. Les conceptions numériques permettent un gain de temps et une réduction des coûts par l'élimination des phases prototypes, à condition que les modèles utilisés soient pourvus de données d'entrée fiables et validées. Les lois de comportement dynamique des matériaux utilisées dans un contexte de sollicitation au crash sont issues du protocole établi par Visteon Systèmes Intérieurs [2,3]. Travaillant sur une étude simplifiée, le modèle complet de sac est remplacé par une membrane dans laquelle une pression isobarique est appliquée. La loi de pression utilisée provient de relevés expérimentaux sur lesquels un facteur majorant de sécurité a été introduit. De cette façon, le modèle initial a été validé par comparatif vidéométrique, assurant ainsi le caractère prédictif indispensable à toute simulation numérique. Le modèle de 


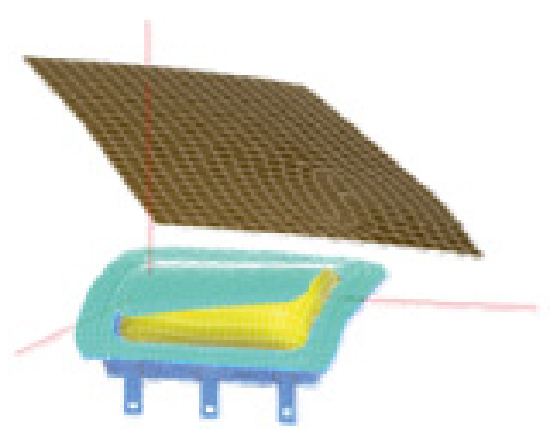

(a)

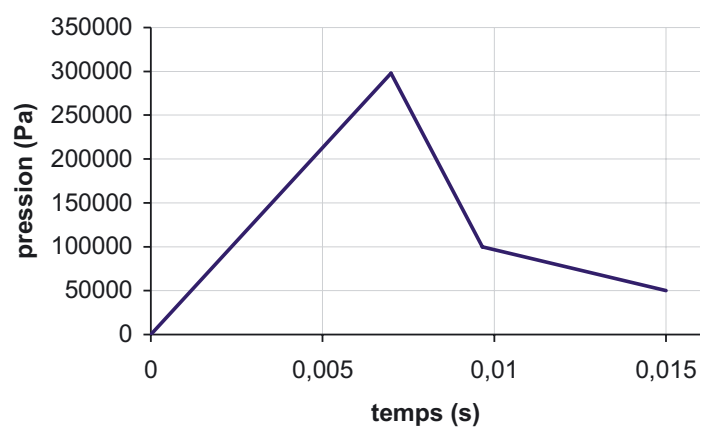

(b)

Fig. 4. Modélisation de référence. a) Visualisation du modèle éléments-finis. b) Loi de pression appliquée au sac simplifié.

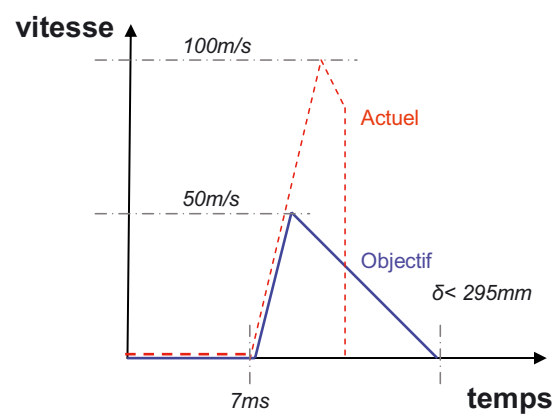

Fig. 5. Profils de vitesse en extrémité de volet en fonction du temps.

la figure 4 représente la zone airbag passager avant. Il est constitué des deux renforts en PP chargé $30 \%$ fibres de verre, de la charnière métallique, et d'un sac simplifié. Le renfort est encastré en son pourtour afin de n'extraire de conclusions relativement qu'à la géométrie de la charnière.

Le profil de vitesse tangentielle en extrémité de volet du modèle de référence est représenté sur la figure 5 .

Nous cherchons à définir une courbe dite «idéale » de ce profil de vitesse afin d'éviter le contact volet/parebrise. Le temps d'ouverture d'environ 7 ms est défini dans le cahier des charges du constructeur, et nous nous donnons pour objectif de diviser le pic de vitesse par 2, soit $50 \mathrm{~m} . \mathrm{s}^{-1}$ au maximum, comme schématisé sur la figure 5 . Il s'agit donc de procurer la même accélération du volet dans les premiers instants afin de libérer l'ouverture

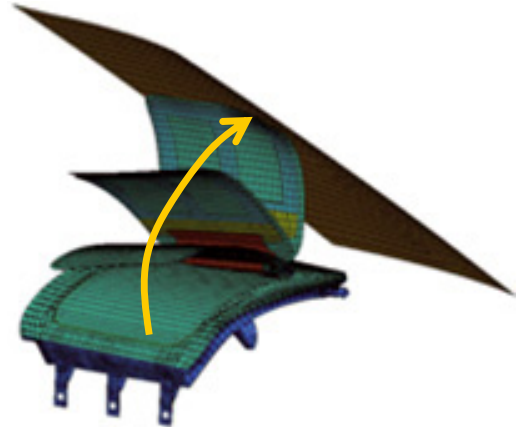

(a)

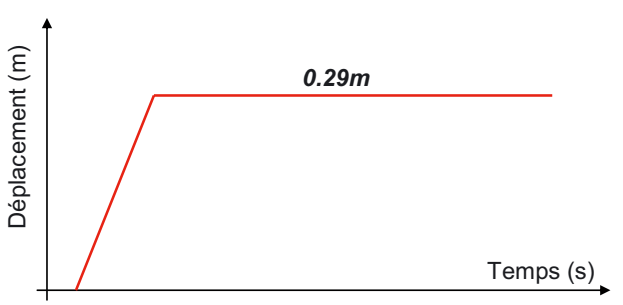

(b)

Fig. 6. Visualisation de la développante maximale autorisée. a) Développante du volet. b) Profil de déplacement limite.

nécessaire au déploiement du sac, puis de ralentir le volet dès qu'il atteint un angle d'ouverture de $45^{\circ}$ avec la normale à la planche de bord. Ensuite, on définit la droite de vitesse permettant un ralentissement du volet permettant d'atteindre une vitesse nulle avant contact avec le parebrise. L'instant $t$ du contact est défini par la développante du volet par rapport à l'axe de la charnière, ce qui régit la pente de ralentissement, représentée sur la figure 6 .

Grâce au premier calcul, nous avons pu déterminer que la zone qui impacte en premier le pare-brise doit parcourir $0,295 \mathrm{~m}$. L'étude se résume donc à vérifier la relation suivante (Eq. (1)) :

$$
\int_{t=0}^{t=t(v=0)}\left|V_{\text {noeud }} \cdot \mathrm{d} t\right|\langle 0,29
$$

avec $V_{\text {noeud }}$ la vitesse du nœud central situé en extrémité de volet, et $t$ le temps.

Pour atteindre cet objectif, il suffit de parfaire la cinématique d'un nœud de maillage situé en extrémité du volet et d'optimiser sa vitesse au cours du temps par l'intégration de systèmes adéquats.

\section{Pré-étude sur formes simples}

Afin de réduire les temps de développement d'une solution pertinente tout en testant un nombre non limité de géométries de charnière, des simulations simples sont réalisées. Ces petits cas tests permettent de repérer des tendances en termes d'efficacité et de retenue à la flexion. Il s'agit d'une plaque encastrée sur l'une des extrémités et 
Tableau 1. Schématisation des cas testés.

\begin{tabular}{|c|c|c|c|c|c|}
\hline Forme & Plane & Vague U & Triangle & Carrée & Carrée décalée \\
\hline$E(J)$ & 6,35 & 9,13 & 9,18 & 9,39 & 34,46 \\
\hline$V\left(\mathrm{~m} \cdot \mathrm{s}^{-1}\right)$ & 51,25 & 40,75 & 38,99 & 38,84 & 15,52 \\
\hline$T(\mathrm{~ms})$ & 4,5 & 5,2 & 5,2 & 5,3 & 5,8 \\
\hline
\end{tabular}

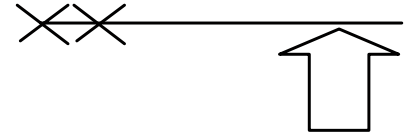

Fig. 7. Schématisation du cas test.

à laquelle un effort de $250 \mathrm{~N}$ est appliqué à son extrémité opposée, selon la configuration représentée sur la figure 7. Cette sollicitation de flexion s'apparente à celle subie par la charnière; une partie plane encastrée et l'autre subissant la pression de l'airbag.

Les résultats les plus pertinents issus des simulations numériques des cas tests sont reportés dans le tableau 1. Celui-ci se lit selon la légende décrite ci-dessous :

- E(J) : énergie dissipée lors de la déformation de la charnière ;

- $V\left(\mathrm{~m} . \mathrm{s}^{-1}\right)$ : vitesse maximale atteinte par l'extrémité de la charnière;

- $T$ (ms) : temps nécessaire pour avoir un angle de $90^{\circ}$ entre les deux plans de la charnière.

La forme plane constitue notre cas de référence et s'affirme comme étant la géométrie la plus défavorable à une sollicitation de flexion. Concernant les formes simples de $\mathrm{U}$, de triangle et de carré, les résultats chiffrés en termes d'énergie absorbée, de pic de vitesse et de delta de temps $\left(0-90^{\circ}\right)$ montrent une similitude parfaite. On retrouve ici la raison pour laquelle la majorité des charnières de volet d'airbag sont conçues en forme de $\mathrm{U}$ ou de double $\mathrm{U}$, les formes carrées et triangles n'apportant aucun avantage technique et présentant une agressivité envers le sac du fait des arêtes vives.

Seule la solution en carrés décalés montre un réel avantage. Celle-ci permet de dissiper 3,7 fois plus d'énergie lors de l'ouverture tout en divisant le pic de vitesse de rotation par 2,5. Cette solution montre également une capacité de freinage importante puisque le temps écoulé pour atteindre une position de $90^{\circ}$ est allongé de $10 \%$.

La conclusion de cette étude témoigne du fait que les solutions présentant un axe de flexion naturel parallèle à l'axe de rotation sont moins efficaces en terme de freinage que les solutions présentant plusieurs axes de flexion asynchrones ou un axe non parallèle à l'axe de rotation.

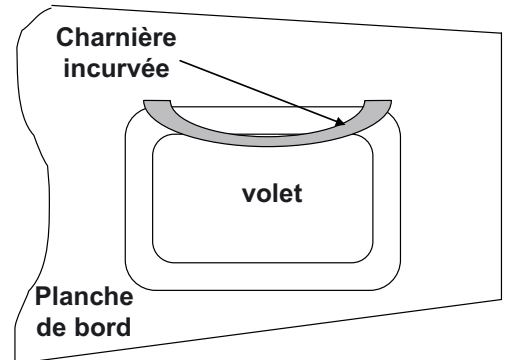

(a)

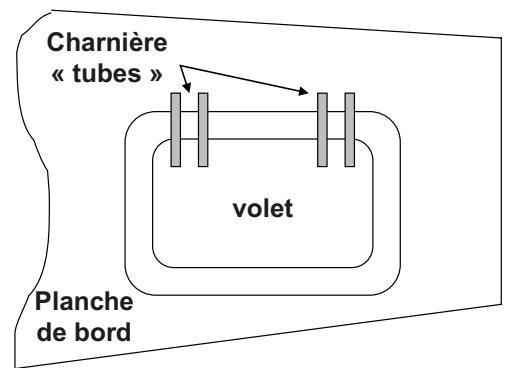

(b)

Fig. 8. Schématisation des charnières dans l'environnement planche de bord. a) Charnière incurvée. b) Charnière à tubes perpendiculaires.

\section{Géometries intégrées}

Suite aux conclusions précédentes, des conceptions de charnière dont l'axe de flexion ne se présente pas parallèlement à l'axe de rotation du volet airbag sont étudiées sur produit. Deux concepts particuliers sont présentés : la charnière incurvée (Fig. 8a) et la charnière composée de tubes perpendiculaires à l'axe de rotation du volet (Fig. 8b). Les schémas de principe sont représentés sur la figure 8 .

La solution a) de la figure 8 a pour objectif de consommer de l'énergie dans la partie arrière de la charnière. En effet, lors de l'ouverture du volet, il est nécessaire de changer le sens de courbure de la tôle, ce qui nécessite une part non négligeable de l'énergie disponible.

La charnière utilisant les tubes positionnés perpendiculairement à l'axe de rotation du volet a pour but de tester une solution extrême; la forme tubulaire étant 


\begin{tabular}{|l|l|l|}
\hline$\left(\mathrm{a}_{1}\right)$ Charnière peu incurvée & $\left(\mathrm{a}_{2}\right)$ Charnière incurvée & $\left(\mathrm{a}_{3}\right)$ Charnière très incurvée \\
\hline & & \\
\hline
\end{tabular}

Fig. 9. Visualisation des courbures de charnière testées.

Tableau 2. Résultats numériques selon courbures de charnière.

\begin{tabular}{|l|c|c|}
\hline Type de charnière & $\begin{array}{c}\text { Effort à } \\
\text { l'impact }(\mathrm{N})\end{array}$ & $\begin{array}{c}\text { Temps à } \\
\text { l'impact }(\mathrm{ms})\end{array}$ \\
\hline Initiale en $\mathrm{U}$ & 3860 & 14,2 \\
\hline Peu incurvée $\left(\mathrm{a}_{1}\right)$ & 3300 & 14,5 \\
\hline Incurvée $\left(\mathrm{a}_{2}\right)$ & 3060 & 14,6 \\
\hline Très incurvée $\left(\mathrm{a}_{3}\right)$ & $\mathbf{7 5 5}$ & $\mathbf{1 8 , 4}$ \\
\hline
\end{tabular}

la géométrie présentant la résistance à la flexion la plus importante.

\subsection{Charnière incurvée}

Trois courbures de charnière sont testées afin d'évaluer l'influence de ce dernier paramètre. Les géométries de calcul sont présentées sur la figure 9 .

Le fait d'incurver la charnière simple a permis d'obtenir de bons résultats au niveau de l'effort d'impact entre le pare-brise et le volet. Le tableau 2 montre que l'augmentation de la courbure donne des résultats satisfaisants au niveau de l'impact volet/pare brise.

Nous observons que la diminution du rayon de courbure provoque une augmentation importante de l'énergie consommable par celle-ci. La charnière consommant beaucoup d'énergie dans sa phase de flexion, le volet perd de la vitesse et impacte le pare-brise beaucoup plus tardivement par rapport à une solution classique (Fig. 10).

Néanmoins, ce design présente un inconvénient majeur : la résistance de la charnière se manifeste dès le début de la sollicitation et retarde l'instant de l'ouverture du volet; ce qui s'oppose aux cahiers de charges des constructeurs.

\subsection{Charnière tubulaire}

La charnière est constituée de quatre tubes placés perpendiculairement à l'axe de rotation du volet, et répartis deux à deux sur les extrémités latérales afin de garantir la stabilité du volet lors de l'ouverture. Une représentation en éléments-finis est montrée sur la figure 11.

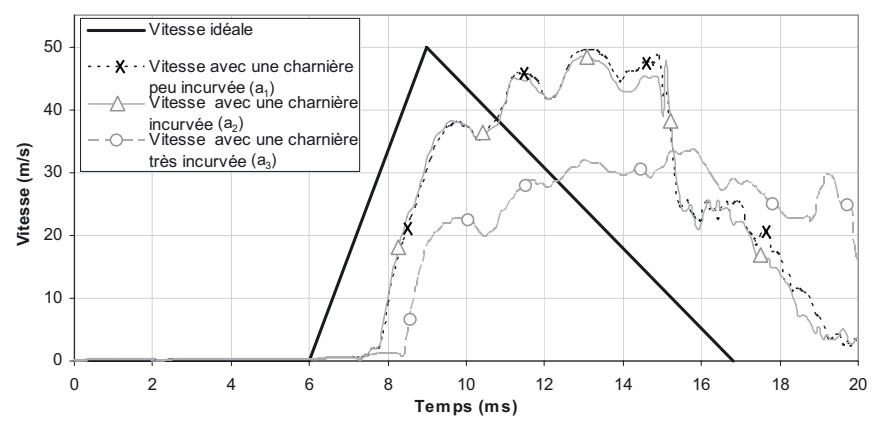

Fig. 10. Comparatif des vitesses à l'extrémité du volet selon courbures de charnière.

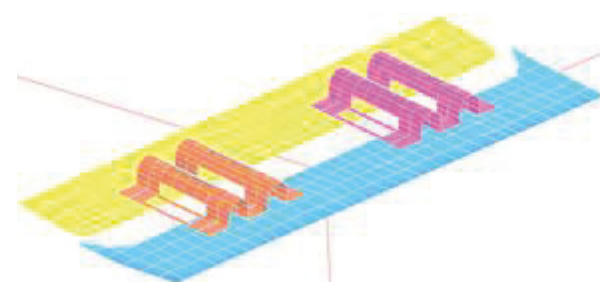

Fig. 11. Visualisation du concept « tube ».

Cette conception permet au volet de s'ouvrir sans impacter le pare-brise. L'objectif principal de notre étude se voit donc concrétisé, mais la capacité à absorber de l'énergie a un coût en terme de contraintes appliquées au renfort. En effet, dans le cas des tubes comparativement à la charnière initiale en $U$, les niveaux de contraintes sont plus importants. Le renfort subit de fortes contraintes dans les coins où est attachée la charnière ainsi que sur les pattes latérales, d'où la nécessité de modification dans la conception des renforts. L'ensemble de ces conclusions est repris sur la figure 12 .

Sur la courbe représentant la distance développée en extrémité de volet, nous remarquons le palier à $0,29 \mathrm{~m}$. Ce palier atteste qu'avec la conception de charnière utilisant des tubes, le volet arrive sur le pare-brise avec une vitesse quasi-nulle. Sur la simulation numérique, le «nœud témoin » du volet reste en contact avec le parebrise, avant un retour élastique global de la structure. Cette solution est proche de nos objectifs. Néanmoins, la géométrie de tube permet de limiter l'accélération du volet post ouverture, amenant ainsi la vitesse tangentielle en extrémité de volet à un maximum de $40 \mathrm{~m} . \mathrm{s}^{-1}$, comparativement à $100 \mathrm{~m} . \mathrm{s}^{-1}$ pour notre cas initial de référence, 


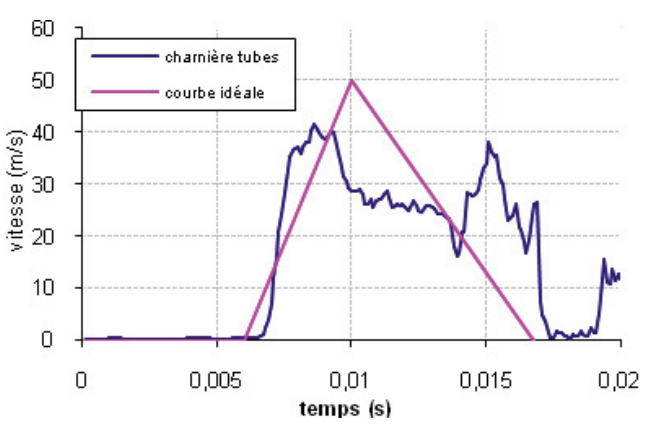

(a)

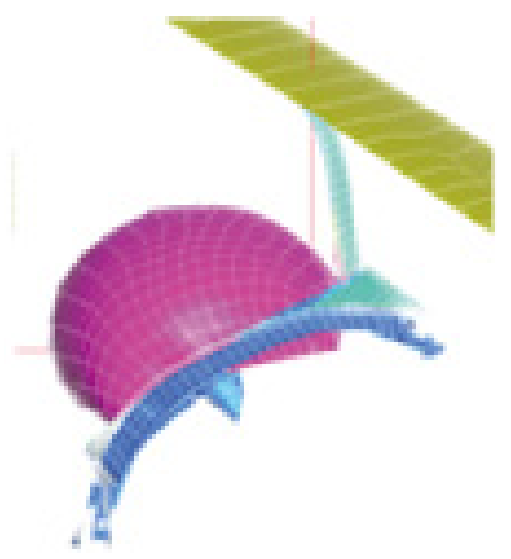

(c)

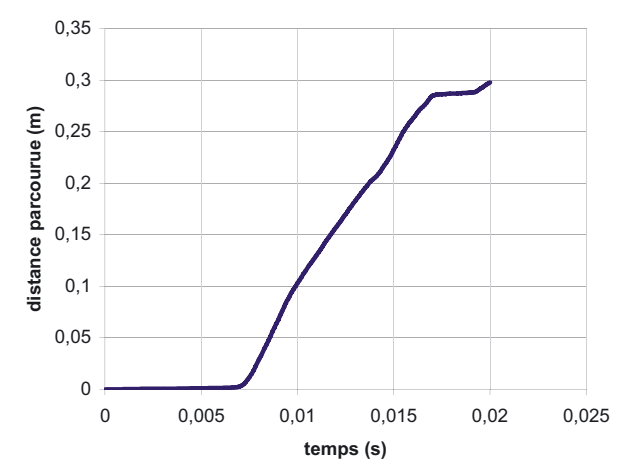

(b)

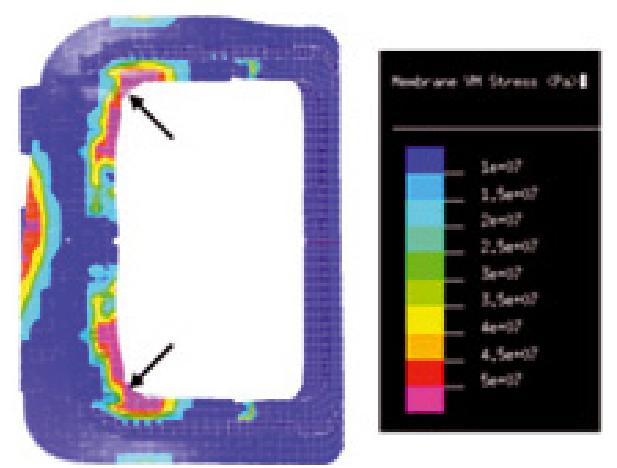

(d)

Fig. 12. Résultats obtenus avec modélisation en charnière «tube ». a) Représentation de la réponse en vitesse de l'extrémité du volet. b) Représentation de la réponse en distance développée de l'extrémité du volet. c) Visualisation de l'angle d'ouverture maximal du volet. d) Visualisation des zones de contrainte sur le renfort.

mais ne permet pas une décélération progressive de celuici une fois la vitesse maximale atteinte. En effet, le second pic observable sur la figure 12a est directement lié à une localisation importante de la déformation plastique des tubes sollicités en flexion.

À $t=13,5 \mathrm{~ms}$, les tubes « charnière » cèdent à la flexion et se croquent, créant ainsi une discontinuité de la déformation plastique, jusqu'alors homogène le long de la génératrice des tubes.

La solution « produit », plus largement discutée dans la section suivante, s'attache à intégrer un centre instantané de rotation (CIR) au cours de l'ouverture du volet, garantissant ainsi une résistance optimale à la flexion, et ne présentant pas de perte de propriétés mécaniques audelà d'un certain angle d'ouverture.

\section{Application produit}

Suite aux simulations numériques précédentes, les conclusions pointent sur les critères importants nécessaires à l'obtention d'une solution technique répondant à nos attentes :

- la forme de la charnière ne doit pas être homogène. En effet, elle doit présenter des zones très rigides permettant au renfort de maintenir un niveau de contrainte acceptable et des zones souples pouvant se plier et consommer l'énergie cinétique du volet;

- éviter des zones figées qui favorisent le freinage jusqu'à une valeur limite d'ouverture volet au-delà de laquelle la charnière ne présente aucune résistance mécanique.

Plusieurs modélisations basées sur des tubes ont permis d'obtenir des résultats probants. Le principe qui consiste à intégrer différents types d'alésages latéraux montre l'avantage de créer artificiellement un CIR qui répartit à chaque instant les contraintes plastiques de façon homogène sur les éléments « charnière ». L'ensemble de cette démarche a conduit au développement d'une géométrie de cônes « charnière », comme présenté sur la figure 13 .

- La zone 1 permet la fixation au renfort de planche de bord et la répartition des efforts sur une surface de contact importante.

- La zone 2 comporte des raidisseurs augmentant la résistance à la flexion, ce qui permet de contenir les niveaux de contraintes par une limitation à la déformation. 


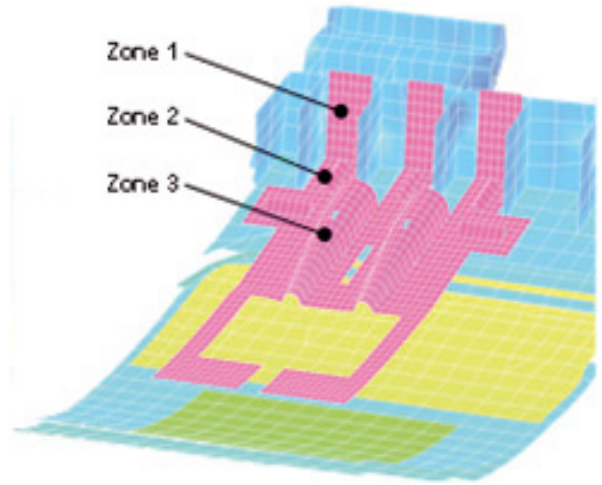

Fig. 13. Visualisation de la conception « charnière cône ».

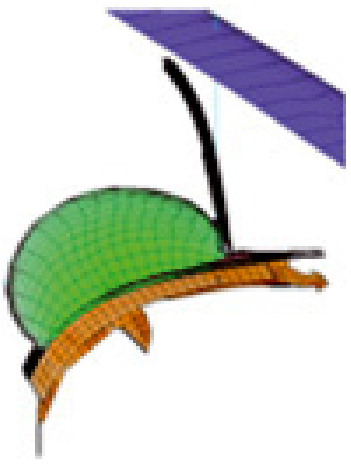

(a)

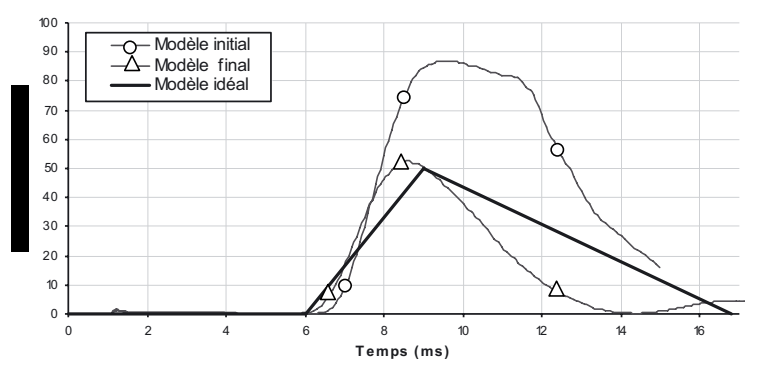

(b)

Fig. 14. Visualisation des résultats obtenus par le concept «cône ». a) Visualisation de l'ouverture maximale du volet. b) Comparatif de l'énergie cinétique produite par le volet entre modèle optimisé et modèle référence.

- La zone 3 est la zone de fonctionnalité de la charnière. La forme conique est responsable du CIR, et l'alésage central est l'initiateur des déformations plastiques.

En plus de l'efficacité démontrée de cette solution, celle-ci offre l'avantage de ne présenter aucune agressivité envers le sac : ni arête vive, ni point d'accroche empêchant le déploiement du sac. Cette conception de charnière garantit la principale spécification client puisqu'elle absorbe suffisamment d'énergie pour éviter le contact entre le volet et le pare-brise. Le volet est parfaitement maintenu tout au long de l'ouverture et les temps d'ouverture et pente d'accélération initiale ne sont pas dégradés, comme le montre la figure 14 .

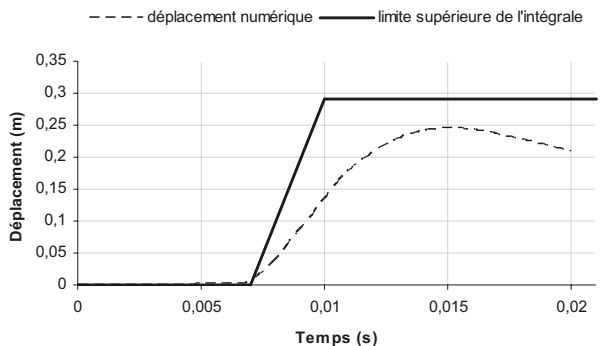

Fig. 15. Visualisation de la développée en extrémité de volet.

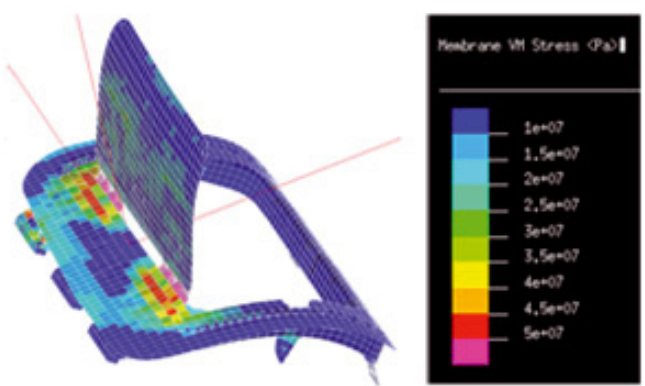

Fig. 16. Visualisation des contraintes sur le PP renforcé en position d'ouverture volet maximale.

La courbe de la figure 14 montre un comparatif de l'énergie cinétique développée par le volet entre le modèle initial et la solution optimisée. À $t=8,5 \mathrm{~ms}$, nous voyons que la décélération du volet intervient au niveau du volet intégrant la solution optimisée, ce qui permet de réduire de $35 \%$ l'énergie cinétique du volet.

En terme de déplacement, en comparant la courbe numérique et la courbe analytique représentant la borne supérieure de l'intégrale (Eq. (1)), représentées sur la figure 15, nous observons la validité de la solution par rapport à notre premier critère.

En terme de conception mécanique « produit », les niveaux de contrainte atteints dans le renfort sont réduits de façon conséquente puisque les valeurs maximales atteignent 40 à $50 \mathrm{MPa}$, ce qui représente une valeur acceptable pour le polypropylène spécifique sollicité à haute vitesse de déformation plastique. Sur la figure 16, nous pouvons également remarquer que l'étendue des zones sollicitées a été réduite, ce qui atteste d'une meilleure répartition des efforts à travers l'ensemble des éléments constitutifs de la planche de bord.

\section{Validation physique}

L'ensemble de ces conclusions numériques attestant de la viabilité du concept de charnière de forme conique, la réalisation de charnières prototypes est engagée selon la définition de forme numérique afin de valider expérimentalement cette conception. Les charnières en acier sont obtenues par emboutissage et usinage, et deux éléments de charnières d'épaisseur $0,7 \mathrm{~mm}$ sont intégrés à la zone airbag de notre planche de bord, comme le montre la figure suivante. La planche de bord faisant objet 


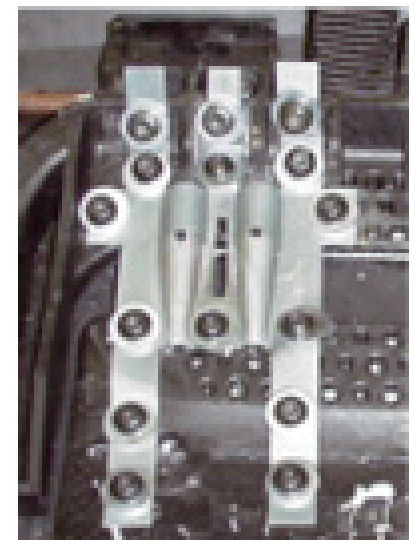

Fig. 17. Visualisation de l'intégration de la charnière prototype sur la planche de bord série.

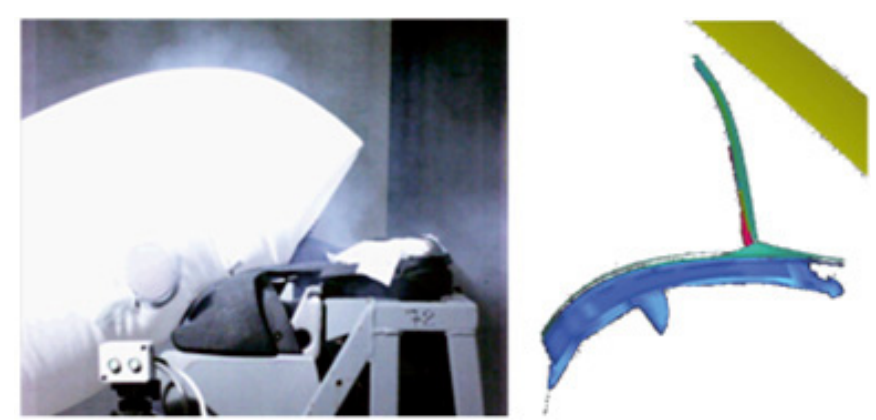

Fig. 18. Comparatif de l'angle maximal d'ouverture volet entre essai expérimental et simulation numérique.

de notre étude n'étant pas initialement dimensionnée et conçue pour recevoir ce type de charnière, le mode de fixation prototype, représenté sur la figure 17, est réalisé par vissage, ce qui affaiblit légèrement les pattes de fixation des charnières et fragilise la planche de bord elle-même.

L'essai de tir airbag est réalisé à l'aide d'un module airbag série, à la température de $23{ }^{\circ} \mathrm{C}$, et sans parebrise. L'ensemble est filmé par deux caméras de fréquence d'échantillonnage $1000 \mathrm{im} . \mathrm{s}^{-1}$, dont l'une est positionnée latéralement afin de quantifier l'angle maximal atteint par le volet.

Suite aux essais expérimentaux, un angle d'ouverture maximal de $130^{\circ}$ est observé, comparativement à un angle de $90^{\circ}$ calculé numériquement, ce qui conduit à un impact pare-brise certain. Un comparatif entre la prédiction numérique et le résultat expérimental est présenté sur la figure 18.

Cet écart s'explique, sur la figure 19, par un déchirement des charnières le long des pattes de fixation latérales externes en zone 2. Les congés, de rayon trop faible, n'ont pas permis d'éviter la concentration de contraintes dans les angles. Ces congés n'étant pas définis numériquement pour des raisons de taille d'éléments impliquant des temps de calcul trop importants, la pièce expérimentale a été dimensionnée par défaut.

Supposant que les charnières soient renforcées aux endroits des casses, elles auraient davantage participé à

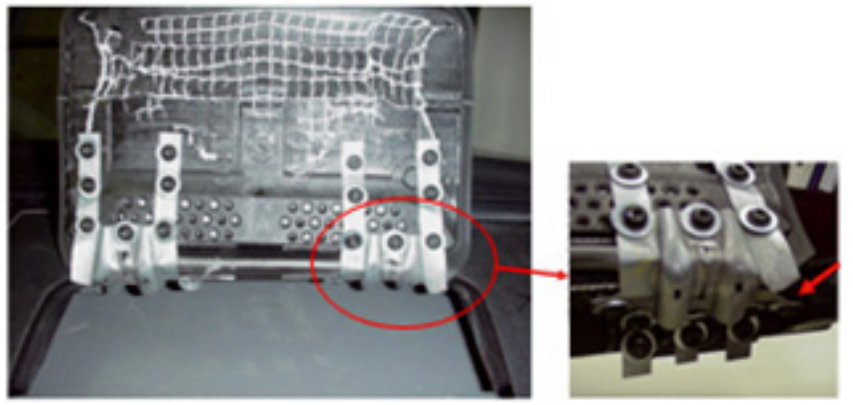

Fig. 19. Visualisation des déchirements latéraux des charnières en zone 2 .

l'absorption de l'énergie développée lors de l'ouverture $\mathrm{du}$ volet et auraient permis de stopper le volet à un angle se rapprochant des valeurs calculées par simulation numérique ne prenant pas les phénomènes de rupture en compte.

En effet, lors de la rupture de certaines zones métalliques sollicitées, une partie de l'énergie est absorbée mais la ruine de la charnière mène rapidement à la fragilisation du concept frein qui n'offre plus la résistance calculée.

Au niveau de la corrélation essai expérimental/ simulation numérique, nous observons lors des tirs de nombreuses similitudes dans le comportement des charnières.

En dehors de la rupture des pattes extérieures non représentées en simulation numérique, car non prises en compte, les déformées constatées expérimentalement sont très proches de celles calculées numériquement, comme le montre la figure 20. Par exemple, nous pouvons remarquer l'incurvation vers l'intérieur des cônes à l'endroit où se produit le pliage.

\section{Conclusion}

L'étude communiquée s'est intéressée à la limitation de la vitesse des volets d'airbag passager de planche de bord, et plus particulièrement à l'impact de celui-ci contre le pare-brise. Le choc entre pièces est discriminant envers la notion de sécurité passive, car le risque de projection de parties de pièces et de matériaux ne peut être évité.

Visteon Systèmes Intérieurs démontre, à travers cet article, sa capacité de conception de structures fiables et répondant aux attentes des clients, par l'intégration croissante de la simulation numérique. L'étude, menée sur une planche de bord série non dimensionnée pour recevoir la géométrie de charnière optimisée, a débouché sur une validation expérimentale correcte. En effet, les déformées des charnières après essais sont similaires à celles prédites par le code de calcul Pam-Crash ${ }^{T M}$, et l'angle de $130^{\circ}$ effectué par le volet en essai est proche de celui calculé numériquement $\left(90^{\circ}\right)$. Cet écart s'explique à la fois par l'amorce de rupture présente sur les côtés externes des charnières, ce qui provoque un assouplissement de la liaison, et par le modèle d'airbag très simplifié dans la mise en donnée du code de calcul. En fait, ce modèle ne reproduit pas la physique expérimentale en terme de temps de 

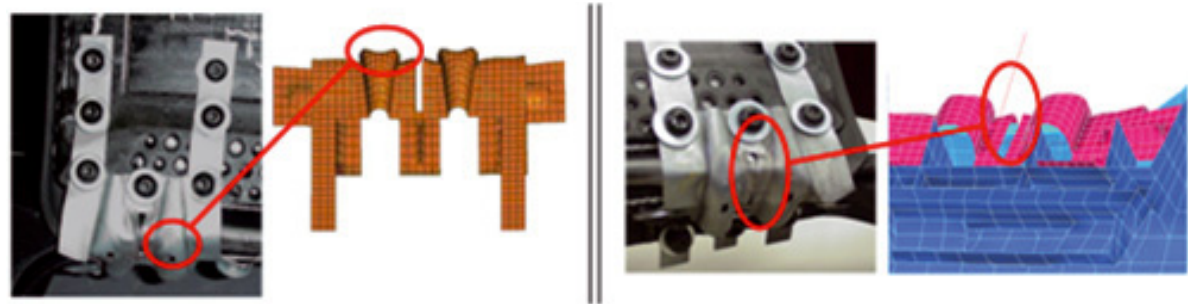

Fig. 20. Similitudes entre simulation numérique et essais expérimentaux.

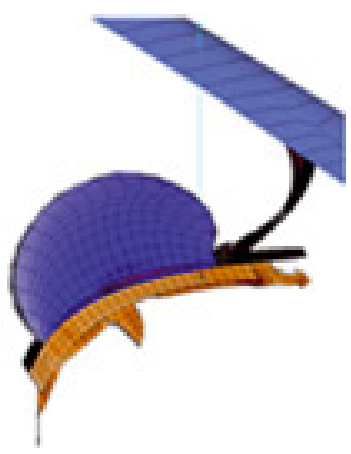

(a)

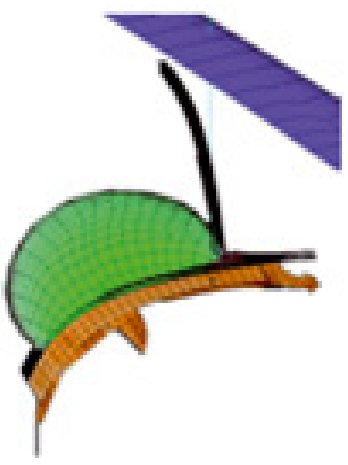

(b)

Fig. 21. Visualisation du bénéfice de la solution optimisée. a) Modèle initial. b) Modèle optimisé.

contact entre sac et volet, et la vidéo démontre un temps de contact plus important lors des essais.

Aussi, comparativement à la charnière initiale, le concept permet d'éviter le contact avec le pare-brise moyennant un dimensionnement adapté au niveau des renforts soudés de planche de bord, comme présenté sur la figure 21 .

Enfin, cette solution présentant une avance technologique en terme de sécurité passive, Visteon Systèmes Intérieurs dispose d'un brevet [4] regroupant un ensemble de concepts de charnières dont l'axe de flexion n'est pas parallèle à l'axe de rotation du volet de planche de bord.

En conclusion, une application produit est présentée sur la figure 22. Cette conception intègre les trois fonctions discutées en introduction, à savoir, le dégagement du volet, la rotation de celui-ci, puis la fonction frein, et permet, par la technique, d'atteindre les spécifications
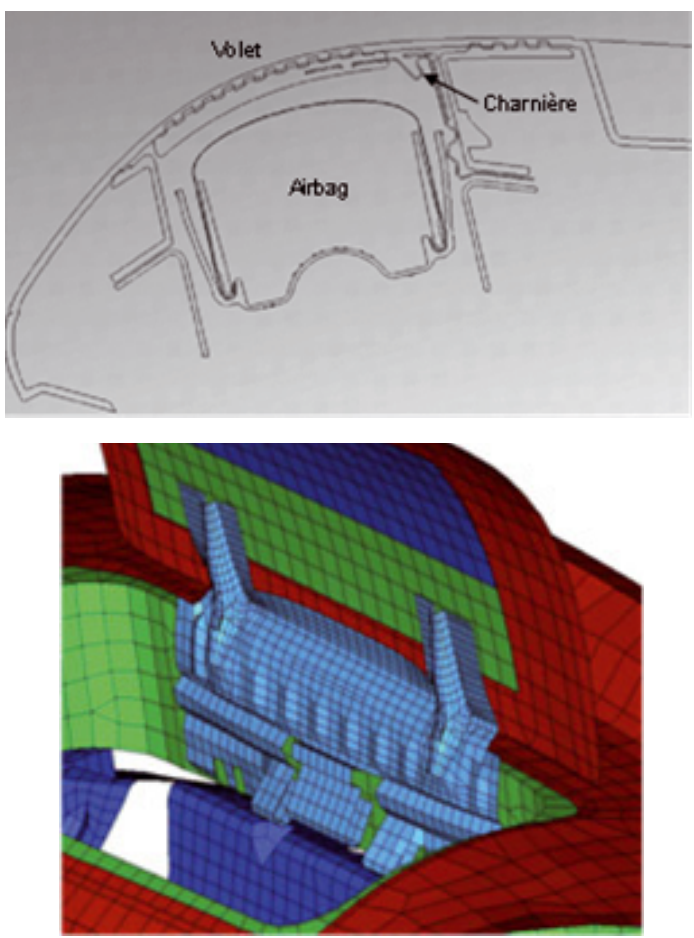

Fig. 22. Visualisation de la solution produit.

du constructeur, objectif quotidien de Visteon Systèmes Intérieurs.

\section{Références}

[1] E. Haug, D. Ulrich, The PAM-CRASH ${ }^{T M}$ Code as an Efficient Tool for Crashworthiness Simulation and Design, 2nd European Cars/Trucks Simulation Symposium, Munich, Germany, May, 1989

[2] G. Spingler, Numerical Modelling of Polymer Materials for Crash Applications, NAFEMS World Congress 2005, Auto Applications 3, 2005

[3] G. Spingler, P. Drazetic, E. Markiewicz, Dynamic Characterization of Polymers to Improve Numerical Simulations for Passive Safety, Int. J.Crashworthiness 10 (2005) 87-10

[4] G. Spingler, Ch. Bey, Visteon Global Technologies, Inc., Dispositif d'articulation formant charnière avec limitation de mouvement et arrangement de coussin gonflable le comprenant, République Française, Institut National de la Propriété Industrielle, Numéro d'enregistrement national 03 06807, 2003 